Faculty of Mathematical Sciences

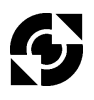

University of Twente

The Netherlands
P.O. Box 217

7500 AE Enschede

The Netherlands

Phone: +31-53-4893400

Fax: +31-53-4893114

Email: memo@math.utwente.nl

www.math.utwente. $\mathrm{nl} /$ publications

Memorandum No. 1627

Shapley value for constant-sum games

A.B. KhmelnitskayA

JunE, 2002

ISSN 0169-2690 


\title{
Shapley value for constant-sum games ${ }^{\star}$
}

\author{
Anna B. Khmelnitskaya ${ }^{\star \star}$ \\ Faculty of Mathematical Sciences, University of Twente, \\ P.O. Box 217, 7500 AE Enschede, The Netherlands
}

\begin{abstract}
It is proved that Young's [2] axiomatization for the Shapley value by marginalism, efficiency, and symmetry is still valid for the Shapley value defined on the class of nonnegative constant-sum games and on the entire class of constant-sum games as well. To support an interest to study the class of nonnegative constant-sum games we show that such a game appears under reasonable reconsideration of the initial TU game before applying one or another solution concept.
\end{abstract}

Keywords: cooperative TU game, value, axiomatic characterization, Shapley value.

Mathematics Subject Classification 2000: 91A12

\footnotetext{
* The research was partially done during the author's February 17-27, 2002, stay at the University of Twente at Enschede, being on leave from SPb Institute for Economics and Mathematics Russian Academy of Sciences, 1 Tchaikovsky St., 191187 St.Petersburg, Russia, e-mail: anna@AK3141.spb.edu. The overall research was done in line with research cooperation between Russian Federation and The Netherlands approved by The Netherlands Organization for Scientific Research (NWO) (Research grant corr.nr. 2000/4/ib, dos.nr. 047-008-010).

** I am grateful to Theo Driessen, Natalia Naumova and Elena Yanovskaya for interesting discussions and comments.
} 
The main problem of cooperative game theory is to find a fair solution how to distribute the total surplus or overall savings among all participants. According to various combinations of different principles of fair division different solution concepts (Shapley value, Banzhaf value, random-order values, core, nucleolus, etc.) are introduced and studied. However, in many real situations it seems reasonable first to reconsider the initial game and only then to apply one or another solution to the new appeared re-estimated game. Indeed, a cooperative game with transferable utility (TU game) is a pair $\langle N, v\rangle$, where $N=\{1, \ldots, n\}$ is a finite set of $n \geq 2$ players and $v: 2^{N} \rightarrow \mathbb{R}$ is a characteristic function, defined on the power set of $N$, satisfying $v(\emptyset)=0$. A subset $S \subseteq N$ (or $S \in 2^{N}$ ) of $s$ players is called a coalition, and the associated real number $v(S)$ presents the worth of the coalition $S$, i.e., the gain that the coalition $S$ can guarantee to obtain itself. But $v(S)$ is a self-evaluation of the coalition $S$ done on a stage of formation of a game $\langle N, v\rangle$, before the game $\langle N, v\rangle$ is already formed. Cooperating with other players of $N$ and sharing together with them the total gain of the amount $v(N)$ the members of $S$ may expect their share to be different.

In what follows the set of all games with a fixed player set $N$ we denote $\mathcal{G}_{N}$. For simplicity of notation and if no ambiguity appears, we shall write $v$ instead of $\langle N, v\rangle$ when refer to a game. Throughout the remainder of the paper we restrict our consideration to nonnegative games of the class

$$
\mathcal{G}_{N}^{+}=\left\{v \in \mathcal{G}_{N} \mid v(S) \geq 0, v(S)+v(N \backslash S) \neq 0, \quad \text { for all } S \subseteq N\right\} .
$$

With every game $v \in \mathcal{G}_{N}^{+}$we associate its so-called constant-sum normalization $v^{c} \in \mathcal{G}_{N}^{+}$defined to be

$$
v^{c}(S)=\frac{v(S)}{v(S)+v(N \backslash S)} v(N), \quad \text { for all } S \subseteq N .
$$

Observe that, for all games $v \in \mathcal{G}_{N}^{+}$

(i) $v^{c}$ is a constant-sum game, i.e.,

$$
v^{c}(S)+v^{c}(N \backslash S)=v^{c}(N)=v(N), \quad \text { for all } S \subseteq N ;
$$

(ii) $v^{c}$ is invariant under $c$-normalization, i.e.,

$$
\left(v^{c}\right)^{c}(S)=v^{c}(S), \quad \text { for all } S \subseteq N ;
$$

note that $v^{c}=v$ whenever the initial game $v$ is a constant-sum game itself (e.g., for additive games);

(iii) for any coalition $S \subseteq N$ and its complement $N \backslash S$, the proportionality rule applied to their two-person subgame of the initial game agrees with the standard solution applied to their two-person subgame of its constant-sum normalization $v^{c}$, i.e.,

$$
\frac{v(S)}{v(S)+v(N \backslash S)} v(N)=v^{c}(S)+\frac{v^{c}(N)-v^{c}(S)-v^{c}(N \backslash S)}{2} .
$$


It seems to be rather natural if in a game $v \in \mathcal{G}_{N}^{+}$players of $S$ having in mind the rest of the society $N \backslash S$ would like to re-estimate the initial self-evaluation $v(S)$ and to assume their real worth to be equal to their quota in the total gain of $v(N)$ shared in proportion to starting values of $v(S)$ and $v(N \backslash S)$, i.e., $v^{c}(S)$ that for instance in case of a superadditive game $v$ is certainly not worse than $v(S)$. If we accept the transformation procedure that replaces a game $v \in \mathcal{G}_{N}^{+}$ by its constant-sum normalization game $v^{c}$ we reduce consideration to the class of nonnegative constant-sum games

$$
\mathcal{G}_{N}^{+c}=\left\{v \in \mathcal{G}_{N} \mid v(N) \neq 0, v(S) \geq 0, v(S)+v(N \backslash S)=v(N), \text { for all } S \subseteq N\right\},
$$

and will apply one or another solution already to games $v \in \mathcal{G}_{N}^{+c}$.

It is worth noting that such an approach is not that harmless since it leads to loss of some information. Indeed, the replacement of a game $v \in \mathcal{G}_{N}^{+}$by the game $v^{c} \in \mathcal{G}_{N}^{+c}$ in fact is a factorization of the space $\mathcal{G}_{N}^{+}$by classes of games with the same worth of grand coalition $v(N)$ and the same relative worths of all complementary nonempty coalitions, i.e., the same ratios $\{v(S) / v(N \backslash S)\} \underset{\substack{S \subset N \\ S \neq N \\ S \neq \emptyset}}{ }$.

Remark. The suggested re-estimation of a game $v \in \mathcal{G}_{N}^{+}$by means of $v^{c} \in \mathcal{G}_{N}^{+c}$ is not the unique one. In our approach we simplify the case and consider the rest of the society $N \backslash S$ as a single unit but it might be partitioned into subcoalitions as well and different partitions of $N \backslash S$ also can be taken into account. Other options exist too. For instance, a game $v$ might be replaced by its dual one, monotonic cover, superadditive cover, balanced cover, and so on. However, we shall not discuss the problem more, this topic lays out of the paper's frame.

In what follows we consider only single-valued solutions usually called values. For any set of games $\mathcal{G} \subseteq \mathcal{G}_{N}$, a value on $\mathcal{G}$ is a mapping $\xi: \mathcal{G} \rightarrow \mathbb{R}^{n}$ that associates with each game $v \in \mathcal{G}$ a vector $\xi(v) \in \mathbb{R}^{n}$, where the real number $\xi_{i}(v)$ represents the payoff to player $i$ in the game $v$. The most famous value is the Shapley value [1]. Different axiomatizations for the Shapley value defined on the entire space $\mathcal{G}_{N}$ are known. Two main of them are the classical one given by Shapley [1] and that of Young [2]. However, the original Shapley's axiomatization exploits the additivity axiom that being a very beautiful mathematical statement does not express any fairness property. The axiomatization of Young that characterizes the Shapley value by efficiency, symmetry, and marginalism appears to be more attractive since all the axioms present different reasonable properties of fair division. We prove below that Young's axiomatization is still valid for the Shapley value defined on the class of nonnegative constant-sum games $\mathcal{G}_{N}^{+c}$. Our proof strategy by induction is similar to that in Young. The proof of Young is not applicable directly to the considered case since an important moment in his proof is an expansion of a game $v \in \mathcal{G}_{N}$ via unanimity basis $\left\{u_{T}\right\}_{\substack{T \subseteq N \\ T \neq \emptyset}}$ defined as

$$
u_{T}(S)=\left\{\begin{array}{ll}
1, & T \subseteq S, \\
0, & T \nsubseteq S,
\end{array} \quad \text { for all } S \subseteq N,\right.
$$


but unanimity games $u_{T}$, for all nonempty and non-singleton coalitions $T \subseteq N$, do not belong to $\mathcal{G}_{N}^{+c}$.

Before we state the theorem we recall the main axioms we use:

- a value $\xi$ is efficient if, for all $v \in \mathcal{G}$,

$$
\sum_{i \in N} \xi_{i}(v)=v(N)
$$

- a value $\xi$ is symmetric if, for all $v \in \mathcal{G}$, for any permutation $\pi: N \rightarrow N$, and for all $i \in N$,

$$
\xi_{\pi(i)}\left(v^{\pi}\right)=\xi_{i}(v)
$$

where $v^{\pi}(S)=v(\pi(S))$ for all $S \subseteq N, S \neq \emptyset$;

- a value $\xi$ is marginalist if, for all $v \in \mathcal{G}$, for every $i \in N, \xi_{i}(v)$ depends only upon the $i$ th marginal utility vector $\{v(S \cup i)-v(S)\}_{S \subseteq N \backslash i}$, i.e.,

$$
\xi_{i}(v)=\phi_{i}\left(\{v(S \cup i)-v(S)\}_{S \subseteq N \backslash i}\right),
$$

where $\phi_{i}: \mathbb{R}^{2^{n-1}} \rightarrow \mathbb{R}^{1}$.

Theorem 1. The only efficient, symmetric, and marginalist value defined on $\mathcal{G}_{N}^{+c}$ is the Shapley value.

Proof. Every game $v \in \mathcal{G}_{N}^{+c}$, being a constant-sum game, appears to be a selfdual game, i.e., $v=v^{*}$, where for any $v \in \mathcal{G}_{N}$, a dual game $v^{*}$ is defined as

$$
v^{*}(S)=v(N)-v(N \backslash S), \quad \text { for all } S \subseteq N .
$$

For any game $v \in \mathcal{G}_{N}$ presented via unanimity basis $\left\{u_{T}\right\}_{\substack{T \subset N \\ T \neq \emptyset}}$,

$$
v=\sum_{\substack{T \subset N \\ T \neq \emptyset}} \lambda_{T} u_{T}
$$

the dual game $v^{*}$ can be presented via dual unanimity basis $\left\{u_{T}^{*}\right\}_{\substack{T \subset N \\ T \neq \emptyset}}$ with the same set of coefficients $\lambda_{T}, T \subseteq N, T \neq \emptyset$, i.e.,

$$
v^{*}=\sum_{\substack{T \subseteq N \\ T \neq \emptyset}} \lambda_{T} u_{T}^{*},
$$

since it is easy to check that, for any two games $v, v^{\prime} \in \mathcal{G}_{N}$ and any real $\alpha$,

$$
\begin{gathered}
\left(v+v^{\prime}\right)^{*}=v^{*}+v^{\prime *}, \\
(\alpha v)^{*}=\alpha v^{*} .
\end{gathered}
$$


Therefore, every constant-sum game $v \in \mathcal{G}_{N}^{+c}$ being self-dual can be presented as a linear combination

$$
v=\sum_{\substack{T \subseteq N \\ T \neq \emptyset}} \lambda_{T} w_{T}
$$

of games $w_{T}, T \subseteq N, T \neq \emptyset$, where for all $S \subseteq N$,

$$
w_{T}(S)=\frac{u_{T}(S)+u_{T}^{*}(S)}{2}=\left\{\begin{array}{cc}
1, & T \subseteq S, \\
1 / 2, & T \cap S \neq \emptyset, T \nsubseteq S, \\
0, & T \cap S=\emptyset .
\end{array}\right.
$$

For all $T \subseteq N, T \neq \emptyset, w_{T} \in \mathcal{G}_{N}^{+c}$. Similarly to unanimity game $u_{T}$, in any game $w_{T}, T \subseteq N, T \neq \emptyset$, every player $i \notin T$ is a null-player, i.e., all of his marginal contributions are equal to zero. The Shapley values of both games $w_{T}$ and $u_{T}$ coincide, i.e., for every $i \in N$,

$$
S h_{i}\left(w_{T}\right)=S h_{i}\left(u_{T}\right)=\left\{\begin{array}{cc}
1 / t, & i \in T \\
0, & i \notin T .
\end{array}\right.
$$

However, we cannot apply the induction procedure directly to the expansion (1) since for a nonnegative constant-sum game $v \in \mathcal{G}_{N}^{+c}$, not all coefficients $\lambda_{T}$ in (1) are necessarily nonnegative (we can state only that at least one of them is positive), and deletion of a term in (1) may lead out of the class $\mathcal{G}_{N}^{+c}$.

To overcome the problem we consider another than (1) expansion of a game $v \in \mathcal{G}_{N}^{+c}$ via games $w_{T}, T \subseteq N, T \neq \emptyset$. For an expression (1) and each $t=$ $1, \ldots, n$, define

$$
\lambda_{t}=\max \left\{\max _{T:|T|=t} \lambda_{T}, 0\right\}, \quad \text { and } \quad \bar{\lambda}_{T}=\lambda_{t}-\lambda_{T} \geq 0 .
$$

Consider a symmetric game

$$
u=\sum_{t=1}^{n} \lambda_{t} \sum_{\substack{T \subset N \\ T \neq 0 \\|T|=t}} w_{T}
$$

One can easily see that $u \in \mathcal{G}_{N}^{+c}$. Since (1),

$$
v=u-\sum_{\substack{T \subset N \\ T \neq \emptyset}} \bar{\lambda}_{T} w_{T}
$$

Observe that deletion of any term under the summation sign in (2) does not move out of $\mathcal{G}_{N}^{+c}$ since $\bar{\lambda}_{T} \geq 0, T \subseteq N, T \neq \emptyset$. Let now the index $I$ of a game $v \in \mathcal{G}_{N}^{+c}$ be the minimum number of terms under the summation in an expression (2), i.e.,

$$
v=u-\sum_{k=1}^{I} \bar{\lambda}_{T_{k}} w_{T_{k}}
$$


where all $\lambda_{T_{k}} \neq 0$. We proceed the remaining part of the proof by induction on this index $I$.

Let $\xi$ be an efficient, symmetric, and marginalist value on $\mathcal{G}_{N}^{+c}$.

If $I=0$, then $v=u$, and for symmetric game $u$ the result follows directly from efficiency and symmetry assumptions about both values $\xi$ and the Shapley value.

Assume now that $\xi(v)$ is the Shapley value whenever the index of $v \in \mathcal{G}_{N}^{+c}$ is at most $I$, and consider some $v \in \mathcal{G}_{N}^{+c}$ with the index equal to $I+1$. Let $T=\cap_{k=1}^{I+1} T_{k}$. For all $i, j \in T$, symmetry implies that $\xi_{i}(v)=\xi_{j}(v)$; the similar statement is true for the Shapley value too. Hence, combined with the requirement of efficiency (both payoff vectors $\xi(v)$ and $S h(v)$ sum up to $v(N)$ ) it is sufficient to prove that $\xi_{i}(v)=S h_{i}(v)$ when $i \notin T$. Define a game

$$
v^{(i)}=u-\sum_{k: i \in T_{k}} \bar{\lambda}_{T_{k}} w_{T_{k}} .
$$

Obviously, the index of $v^{(i)}$ is at most $I$ and, therefore, by induction hypothesis, $\xi\left(v^{(i)}\right)=S h\left(v^{(i)}\right)$. To complete the proof notice that both $i$ th marginal utility vectors relevant to the games $v$ and $v^{(i)}$ coincide and, so, by marginalism of both values $\xi$ and the Shapley value, $\xi_{i}(v)=\xi_{i}\left(v^{(i)}\right)$ and $S h_{i}(v)=S h_{i}\left(v^{(i)}\right)$.

To conclude with it is reasonable to note that Young's axiomatization is valid as well for the Shapley value defined on the entire class of constant-sum games

$$
\mathcal{G}_{N}^{c}=\left\{v \in \mathcal{G}_{N} \mid v(S)+v(N \backslash S)=v(N), \text { for all } S \subseteq N\right\} .
$$

Indeed, the last statement can be proved by the same way as it was done for the case of the class of nonnegative constant-sum games $\mathcal{G}_{N}^{+c}$ or one can exploit the same proof as in Young but with replacement of unanimity games $u_{T}, T \subseteq N$, $T \neq \emptyset$, via games $w_{T}, T \subseteq N, T \neq \emptyset$.

\section{References}

1. Shapley LS (1953) A value for n-person games. In: Tucker AW, Kuhn HW (eds.) Contributions to the theory of games II. Princeton University Press, Princeton, NJ, pp. 307-317

2. Young HP (1985) Monotonic solutions of cooperative games. Int J Game Theory 14:65-72 